\title{
Health Information and Cancer Screening Differences for Asian Americans
}

\author{
Steven E. Shive ${ }^{1,2}$, Grace X. Ma ${ }^{2}$, Yin Tan ${ }^{2}$, Jamil I. Toubbeh ${ }^{2}$, \\ Lalitha Parameswaran $^{2}$, Joseph Halowich ${ }^{3}$ \\ ${ }^{1}$ East Stroudsburg University \\ ${ }^{2}$ Center for Asian Health, Temple University \\ ${ }^{3}$ Parkside High School
}

\begin{abstract}
This study examined differences between Asian and non-Asian Americans in sources of health information and cancer screening. A nationally representative sample of 6,722 adults in the US, including those who speak, Spanish, Chinese, Vietnamese and Korean was selected. Questionnaire items included demographic variables, health status, cancer screening, health care experiences, health information sources, and use of an interpreter in the patient-provider interaction. Asians used the internet more for health information, preferred a physician from their own race, had friends or relatives and staff persons serve as interpreters, and less likely to call a health care provider and pharmacist, to need an interpreter, have had a complete physical exam, pap test, mammogram, and colorectal exam, within the last two years, than non-Asian Americans. Difficulty speaking with a physician led to 2.2 times greater risk of never getting a physical exam and 4.3 times greater risk of never getting a prostate exam. Development of sources of health information relevant to Asian Americans may enhance their decision making prior to and in conjunction with receiving medical care services.
\end{abstract}

(C) 2006 Californian Journal of Health Promotion. All rights reserved.

Keywords: Asian, Cancer information, Cancer Screening

Healthcare access may be a significant barrier to reducing health disparities among racial/ethnic groups (IOM, 2003). Accessing health care involves access to health information, access to healthcare providers, facilities, to follow treatment regimens, and to engage in more mutual decision making by consumers. In general, Asians (58\%) have a harder time understanding physician provided health information than Whites $(41 \%)$ which may in part explain why Asians are less satisfied with health care (Saha, Arbelaez, \& Cooper, 2003; Saha \& Hickam, 2003;), and health insurance coverage (Haviland, Morales, Reise, \& Hays, 2003), as compared to other ethnic/racial groups. Access to health information from the World Wide Web, printed materials, or obtaining it from physicians show no racial/ethnic differences (National Health Care Disparities Report, 2003; Williams et al., 1995). Language and underinsurance are barriers to health care access which often cause complications
(Burstin, Lipsitz, \& Brennan, 1992; Gandhi, Burstin, Cook, Puopolo, Haas, Brennan, Bates, 2000).

\section{Language}

Asians who speak little or no English may encounter barriers to health care. Often research in healthcare excludes non-English speakers (Burstin et al., 1992). Approximately 27\% of Asians reported poor communication with their health care providers compared to $17 \%$ of Whites (National Health Care Disparities Report, 2003), and language concordant patients appear to have better health related quality of life (Seijo, 1991). Nearly $14 \%$ of the US population speaks a language in their homes other than English (1990 Census). Lack of an interpreter is a challenge to health care delivery (Erzinger, 1991; Perez-Stable, NapolesSpringer, \& Miramontes, 1997; Seijo, 1991) and often leads to physicians to use interpreters who are untrained and limited in English language 
skills such as family members or staff from nearby ethnic restaurants (Baker, Parker, Williams, Coates, \& Pitkin, 1996; Ginsberg, Martin, Andrulis, Shaw-Taylor, \& McGregor, 1995). Some Asians prefer trained interpreters to use of family members for privacy, and for sensitive issues prefer same sex interpreters (Ngo-Metzger et al., 2003. Research is necessary on utilization rates of types of interpreters.

\section{Screening}

There is disparity in screening rates between ethnic/racial groups. While cancer is the number one cause of death among Asian women (Chen \& Koh, 1997), they have the lowest screening rates of all ethnic groups in the US (American Cancer Society, 2004; Miller et al., 1996). Asian women are less likely to go for a Pap test $(68.2 \%)$ compared to White women $(83.9 \%)$ (American Cancer Society, 2004), and Asian women are more likely to have never had a Pap test $(21 \%)$ than White women $(5 \%)$ of the White women (Kagawa-Singer \& Pourat, 2000). This disparity exists for other cancers as well. Asian women $(57 \%)$ are less likely to have a mammograms than White women $(72.1 \%)$ (American Cancer Society, 2004), and 30\% of Asian women have never had a mammogram compared with $21 \%$ of White women (Chen, Diamant, Kagawa-Singer, Pourat, \& Wold, 2004; Collins et al., 2002; Sanghavi, 2003). For colon cancer, $14.5 \%$ of the Asians $\geq 50$ years have had Fecal Occult Blood Test (FOBT), whereas among Whites it is $18.3 \%$ and the Endoscopy utilization rates within the past five years is $19.2 \%$ and $31.3 \%$, respectively (American Cancer Society, 2004).

The purpose of this study was to examine differences between Asian and non-Asian Americans in sources of health information and cancer screening.

\section{Methods}

A 96-item interview questionnaire, developed by the Commonwealth Fund in collaboration with Princeton Survey Research Association, was used. A nationally representative sample of 6,722 adults age 18 and older living in the continental United States who spoke English, Spanish, Mandarin or Cantonese, Vietnamese or
Korean was selected. The survey was administered from April to November 2001, and took about 25 minutes to complete. The sample consisted of White, African-American, Hispanic and Asian households (Collins, Hughes, Doty, Ives, Edwards \& Tenney, 2002).

\section{Sample}

The sample was designed to generalize to the U.S. adult population (Collins et al., 2002). A stratified minority sample design was conducted, utilizing a random-digit dialing method. Telephone numbers were drawn disproportionately from area code exchange combinations with higher than average densities of minority households. Non-Asians consisted of Whites, African-Americans, Hispanics, Native Americans, and others not self-reported to be Asians. Asians consisted of Chinese, Vietnamese, and Koreans.

\section{Design}

The questionnaire was pre-tested based on a random digit phone sample of respondents. The final questionnaire was translated into multiple languages: Spanish, Mandarin, Cantonese, Vietnamese and Korean. Up to 20 attempts were made to contact a person at every sampled telephone number. Telephone calls took place at various times of the day and days of the week to maximize the chance of making contact with potential participants. Interviewers randomly selected household members.

Five stages of statistical weighting were employed to assure a racial/ethnic distribution of a disproportionately large sample of African Americans, Latinos, and Asian Americans of the matching the distribution of the U.S. general adult population (Collins et al., 2002). The overall response rate was $54.3 \%$ which was based on the contact, cooperation (initial consent obtained), and completion (initially cooperating and eligible participants) rates.

Questionnaire items included demographic variables, health status, cancer screening, health care experiences, health information sources, and use of an interpreter in the patient-provider interaction. Demographic variables included age, gender, English use as primary language 
spoken at home, employment of participant and spouse (full time, part time, unemployed), education level $(<$ high school, high school, college/graduate, post graduate), income, US as country of origin, and length of time in the US (<5years, $>5$ years)

\section{Sources of Health Information and Interaction with Healthcare Provider}

To determine source of health information, participants reported how often they acquire information from the World Wide Web, books or printed material, health care provider, friends or family, the pharmacist, or a community health fair. The ease (very easy, somewhat easy, not too easy, not at all easy, don't look for quality) of acquiring information about quality of physicians in the community, health insurance plans, and hospitals in the community was assessed. Difficulty reading instructions on prescription bottles, and information in booklets were measured (very easy, somewhat easy, not too easy, not at all easy, don't get any information from physician). Perceptions of health status after going to the hospital or health care provider, difficulty communicating with healthcare provider (always, usually, sometimes, never) and need for an interpreter, preference for a healthcare provider from one's own ethnic/racial group, who serves as the interpreter (health care provider, staff person, friend or relative, trained medical interpreter, or someone else), and the interpreter's usual availability were included.

\section{Cancer Screening}

Participants reported previous cancer screening (pap test, mammogram, colon, prostate) for relevant cancers (<than a year ago, 1-2 years, 35 years, more than 5 years ago, or never). Analysis for the complete physical exam included the entire sample, the pap test included all females, mammograms included females 40 and over, colon screening included those age 50 or above, and for prostate cancer males 40 and older.

\section{Statistical Analysis}

Descriptive statistics were reported for the variables that characterized Asian and nonAsians (SPSS 10.0). Chi-square analysis was conducted to examine the bivariate relationship between the dependent variable of being Asian or non-Asian and the independent variables. The independent variables were sex, age, language spoken at home, participant and spouse employment, education level, income, US as country of origin, length of time in the US, illness due to visits to healthcare provider, need for an interpreter with healthcare provider, availability of interpreter, and preference for having physicians from own race/ethnicity.

An independent t-test was used to determine differences in means for age, health information source, information for quality of doctors, insurance plans, and hospitals in the community, difficulty reading/understanding prescription labels and booklets, frequency of interpreters, understanding a physician with interpreter, and screening for cancers. Odds ratios $(95 \% \mathrm{CI})$ were calculated for each health information source, how easy it was to find information about quality physicians, insurance plans, and hospitals, difficulty reading information or booklets in physicians' offices, difficulty in speaking with a physician, and the need to use an interpreter for a physician.

\section{Results}

\section{Characteristics of the Sample}

There was a significant difference between Asians and non-Asians in age, employment, spouse employment, education, income, US as country of origin, and length of time in the US (Table 1). Asian Americans were significantly younger, had full time employment, had higher education, a higher income, be foreign born, and be in the US less than 5 years, compared to nonAsians. While approximately $90 \%$ of Asians spoke English at home, they were significantly less likely to speak English at home than nonAsians. Among Asians who did not speak English as their primary language at home, Mandarin or Chinese (48.8\%), Korean (21.4\%), and Vietnamese $(20.2 \%)$ were spoken. 
Table 1

Characteristics of the Sample

\begin{tabular}{|c|c|c|c|c|}
\hline & $\begin{array}{c}\text { Total } \\
\mathbf{N}=6722\end{array}$ & $\begin{array}{l}\text { Asians } \\
N=627\end{array}$ & $\begin{array}{c}\text { Non-Asians } \\
\mathbf{N}=6095\end{array}$ & Statistic \\
\hline Age (Mean, SD) & $44.8(18.1)$ & $39.5(16.2)$ & $45.3(18.2)$ & $\mathrm{t}=-8.5^{* * *}$ \\
\hline $18-29$ & 22.4 & 29.5 & 21.7 & \\
\hline $30-39$ & 22.8 & 29.2 & 22.1 & \\
\hline $40-49$ & 20.7 & 21.2 & 20.7 & $\chi^{2}$ \\
\hline $50-64$ & 19.7 & 14.3 & 20.3 & \\
\hline $65+$ & 14.4 & 5.9 & 15.3 & $71.6^{* *}$ \\
\hline Female & 61.2 & 52.0 & 62.1 & $24.4^{* *}$ \\
\hline English primary language spoken at home & 92.8 & 87.8 & 93.3 & $773.8^{* *}$ \\
\hline \multicolumn{5}{|l|}{ Language spoken at home } \\
\hline Mandarin/Chinese & --- & 48.8 & --- & --- \\
\hline Korean & --- & 21.4 & --- & --- \\
\hline Vietnamese & --- & 20.2 & --- & --- \\
\hline \multicolumn{5}{|l|}{ Employment } \\
\hline Full-Time & 47.2 & 58.2 & 46.1 & \\
\hline Part-Time & 3.6 & 1.8 & 3.8 & \\
\hline Unemployed & 49.2 & 40.1 & 50.1 & $35.5^{* *}$ \\
\hline \multicolumn{5}{|l|}{ Spouse Employment } \\
\hline Full-Time & 67.0 & 73.5 & 66.2 & \\
\hline Part-Time & 7.9 & 4.8 & 8.2 & \\
\hline Unemployed & 25.1 & 21.7 & 25.5 & $9.6^{*}$ \\
\hline \multicolumn{5}{|l|}{ Student } \\
\hline \multicolumn{5}{|l|}{ Education } \\
\hline$<$ H.S. & 12.5 & 4.7 & 13.3 & \\
\hline High School & 26.0 & 10.9 & 27.5 & \\
\hline College/college graduate & 48.7 & 60.6 & 47.5 & \\
\hline Post Graduate/professional Ed & 12.8 & 23.8 & 11.7 & $177.5^{* *}$ \\
\hline \multicolumn{5}{|l|}{ Income } \\
\hline$<20,000$ & 21.8 & 15.6 & 22.5 & \\
\hline 20K-34,999 & 22.5 & 14.4 & 23.4 & \\
\hline $35 \mathrm{~K}-49,999$ & 18.0 & 19.3 & 17.9 & \\
\hline $50 \mathrm{~K}-74,999$ & 16.2 & 18.3 & 15.9 & \\
\hline $75 \mathrm{~K}+$ & 21.4 & 32.4 & 20.3 & $60.2^{* *}$ \\
\hline US country of origin & 77.3 & 20.4 & 83.1 & $1253.4^{* *}$ \\
\hline \multicolumn{5}{|l|}{ Length of time in US } \\
\hline$<5$ years & 15.7 & 19.9 & 13.6 & \\
\hline$>5$ years & 84.3 & 80.1 & 86.4 & $9.8^{*}$ \\
\hline
\end{tabular}

\section{Sources of Health Information}

As shown in Table 2, Asians were significantly more likely to use the internet as a source of health information than non-Asians, $\mathrm{t}(66)=$ $3.06, \mathrm{p}<.01$. Asians were significantly less likely to call a doctor or health care provider, $\mathrm{t}(66)=$
2.59, $\mathrm{p}<.01$ and pharmacist, $\mathrm{t}(785)=5.77, \mathrm{p}$ $<.001$, to think it was easy to get information about the quality of physicians, $t(5865)=5.93, \mathrm{p}$ $<.001$, health insurance plans, $\mathrm{t}(689)=5.92$, $\mathrm{p}<.001$ and hospitals in the community, $\mathrm{t}(5633)$ $=7.95, \mathrm{p}<.001$, and found significantly greater 
difficulty in reading or understanding information on the prescription bottles, $\mathrm{t}(710)=$ $6.17, \mathrm{p}<.001$ and information booklets in physician offices, $\mathrm{t}(6658)=5.32, \mathrm{p}<.001$, than non-Asians.

Table 2

Source of Health Information

\begin{tabular}{|c|c|c|c|c|}
\hline & $\begin{array}{c}\text { Total } \\
\mathrm{N}=6691\end{array}$ & $\begin{array}{l}\text { Asians } \\
\mathbf{N}=627\end{array}$ & $\begin{array}{c}\text { NonAsians } \\
\mathrm{N}=6694\end{array}$ & Statistic \\
\hline Health information source $^{\mathrm{a}}$ & & & & $t$ \\
\hline From World Wide Web & $3.2(1.0)$ & $3.0(1.0)$ & $3.2(1.0)$ & $-3.1^{* *}$ \\
\hline Books or other printed material & $2.5(1.1)$ & $2.5(1.0)$ & $2.5(1.1)$ & 0.2 \\
\hline Called Doctor/other HC provider & $3.0(0.9)$ & $3.1(0.9)$ & $3.0(0.9)$ & $2.6^{* *}$ \\
\hline Asked friends or family & $2.8(1.0)$ & $2.7(1.0)$ & $2.8(1.0)$ & -0.9 \\
\hline Asked Pharmacist & $2.9(1.0)$ & $3.2(0.9)$ & $2.9(1.0)$ & $2.8^{* * *}$ \\
\hline From Community Health Fair & $2.6(0.7)$ & $3.6(0.7)$ & $3.6(0.7)$ & 1.74 \\
\hline \multicolumn{5}{|l|}{ Ease to find information about the quality of: ${ }^{b}$} \\
\hline Doctors in community & $2.1(1.0)$ & $2.3(1.0)$ & $2.1(1.0)$ & $5.9^{* * *}$ \\
\hline Health insurance plans & $2.2(1.1)$ & $2.4(1.0)$ & $2.1(1.1)$ & $5.9^{* * *}$ \\
\hline Hospitals in community & $1.9(1.0)$ & $2.2(1.0)$ & $1.9(1.0)$ & $8.0^{* * *}$ \\
\hline $\begin{array}{l}\text { Difficulty reading/ understanding instructions on } \\
\text { prescription bottle }\end{array}$ & $1.3(0.7)$ & $1.5(0.8)$ & $1.3(0.7)$ & $6.17^{* * *}$ \\
\hline $\begin{array}{l}\text { Difficulty reading/understanding information/booklets at } \\
\text { Dr's office }(33 b)^{c}\end{array}$ & $1.6(0.9)$ & $1.8(1.0)$ & $1.6(1.0)$ & $5.3^{* * *}$ \\
\hline & & & & $\chi^{2}$ \\
\hline $\begin{array}{l}\text { Got sick or sicker as a result of visiting doctor or } \\
\text { hospital }(\%)\end{array}$ & 14.3 & 9.4 & 15.4 & $14.2^{* * *}$ \\
\hline \multicolumn{5}{|l|}{ Due to mistake by the doctor or hospital $(\%)$} \\
\hline Result of mistake(\%) & 51.7 & 42.1 & 52.3 & \\
\hline Very serious problem & 29.1 & 31.6 & 29.0 & \\
\hline Somewhat serious problem & 18.9 & 26.3 & 18.7 & 2.8 \\
\hline \multicolumn{5}{|l|}{ Not too serious problem } \\
\hline \multicolumn{5}{|l|}{ Preference of Physician $(\%)$} \\
\hline Own race/ethnicity & 10.5 & 15.2 & 10.0 & \\
\hline Another race & 1.0 & 1.4 & 1.0 & \\
\hline No preference & 88.5 & 83.3 & 89.0 & $17.9^{* * *}$ \\
\hline \multirow[t]{2}{*}{ Difficulty speaking with Physician $^{\mathrm{d}}$} & $3.3(1.0)$ & $3.3(0.9)$ & $3.3(1.0)$ & $t=-.05$ \\
\hline & & & & $\chi^{2}$ \\
\hline \multirow[t]{2}{*}{ Needed interpreter for Dr. visit } & 56.9 & 31.4 & 61.7 & $11.6^{* * *}$ \\
\hline & & & & $t$ \\
\hline Frequency of interpreter for physician visit ${ }^{\mathrm{d}}$ & $2.2(1.0)$ & $2.5(1.0)$ & $2.1(1.0)$ & 1.1 \\
\hline Who serves as interpreter (\%) & & & & $\chi^{2}$ \\
\hline Health provider & 1.7 & 0 & 1.8 & \\
\hline Staff person & 55.9 & 33.3 & 57.8 & \\
\hline Friend or relative & 39.0 & 66.7 & 36.7 & \\
\hline Trained medical interpreter & 0.8 & 0 & 0.9 & \\
\hline Someone else & 2.5 & 0 & 2.8 & 3.3 \\
\hline Person usually available to help & 70.1 & 37.5 & 72.5 & $4.3^{*}$ \\
\hline
\end{tabular}

${ }^{\text {a }}$ Where, $1=$ very often, $2=$ somewhat often, $3=$ not too often, $4=$ not at all

${ }^{\mathrm{b}}$ Where $1=$ very easy, $2=$ somewhat easy, $3=$ not too easy, $4=$ not at all easy, $5=$ Don't look for quality

${ }^{\mathrm{c}}$ Where $1=$ very easy, $2=$ somewhat easy, $3=$ not too easy, $4=$ not at all easy, $5=$ Don't get any information

${ }^{\mathrm{d}}$ Where $1=$ always, $2=$ usually, $3=$ sometimes, $4=$ never

$* \mathrm{p}<.05, * * \mathrm{p}<.01, * * * \mathrm{p}<.001$ 
Use of an Interpreter for Understanding a Physician

Asians (15.2\%) preferred to be treated by a racially concordant physician, $\chi 2(3)=17.9, \mathrm{p}$ $<.001$, than non-Asians $(10.0 \%)$ (Table 2). Among participants who had difficulty speaking with a physician, ASIANS (31.4\%) were significantly less likely to need an interpreter than Non-Asians $(61.7 \%), \chi 2(1)=11.6, \mathrm{p}<.001$. Non-Asians indicated that staff persons $(57.8 \%)$ and friends or relatives $(36.7 \%)$ served as interpreters, whereas, Asians used friends or relatives $(66.7 \%)$ and staff persons $(33.3 \%)$ as interpreters, but not significantly $(p>.05)$. NonAsians were significantly more likely to report that the interpreter was always available, $\chi 2(1)=$ $4.34, \mathrm{p}<.05$, than Asians.

\section{Cancer Screening}

Asians were less likely to have had a complete physical exam, pap tests, mammograms, and screening for colon cancer, within the last two years, than non-Asian Americans (Table 3). Access to health information sources such as the World Wide Web, books, a health care provider and being able to ask a pharmacist significantly $(p<.05)$ increased the likelihood of getting all the screening tests (Table 4). Having friends or family as a source of health information significantly $(p<.05)$ increased the likelihood of getting a physical exam, mammogram, and prostate exam. Having access to a community health fair significantly $(\mathrm{p}<.05)$ increased the likelihood of getting a Pap test, mammogram, and prostate exam. Having access to information about quality of physicians in the community significantly increased the likelihood of getting a physical exam, Pap test, and colon cancer test. If it was easy to get information about health insurance plans it was more likely that participants would get a physical exam, and a colon exam.

Table 3

Cancer Screening

\begin{tabular}{|c|c|c|c|c|}
\hline Cancer Screening & $\begin{array}{c}\text { Total } \\
\mathbf{N = 6 6 4 3 ,} \mathbf{\%}\end{array}$ & $\begin{array}{c}\text { Asians } \\
\mathbf{N = 6 2 0 ,} \%\end{array}$ & $\begin{array}{c}\text { NonAsians } \\
\mathbf{N =}=\mathbf{6 0 2 3}, \mathbf{\%}\end{array}$ & $\chi^{\mathbf{2}}$ \\
\hline Complete physical exam & & & & \\
\hline$<1$ year ago & 47.9 & 36.6 & 49.0 & \\
\hline 1-2 years ago & 27.4 & 33.4 & 26.7 & \\
\hline 3-5 years ago & 11.9 & 13.2 & 11.7 & \\
\hline$>5$ years ago & 9.6 & 8.4 & 9.8 & \\
\hline Never & 3.3 & 8.4 & 2.7 & $83.3^{* *}$ \\
\hline Pap test & & & & \\
\hline$<1$ year ago & 56.5 & 47.5 & 57.2 & \\
\hline $1-2$ years ago & 26.5 & 29.4 & 26.3 & \\
\hline 3-5 years ago & 6.9 & 8.3 & 6.8 & \\
\hline$>5$ years ago & 6.3 & 4.6 & 6.4 & \\
\hline Never & 3.8 & 10.2 & 3.3 & $43.4^{* *}$ \\
\hline Mammogram & & & & \\
\hline$<1$ year ago & 49.6 & 40.0 & 50.3 & \\
\hline 1-2 years ago & 28.6 & 33.6 & 28.3 & \\
\hline 3-5 years ago & 6.6 & 5.0 & 6.8 & \\
\hline$>5$ years ago & 4.9 & 5.7 & 4.9 & \\
\hline Never & 10.2 & 15.7 & 9.8 & $9.36^{*}$ \\
\hline Screening for colon cancer & $\mathrm{N}=2196$ & $\mathrm{~N}=120$ & $\mathrm{~N}=2076$ & \\
\hline$<1$ year ago & 26.6 & 19.2 & 27.0 & \\
\hline 1-2 years ago & 22.2 & 17.5 & 22.5 & \\
\hline 3-5 years ago & 11.2 & 13.3 & 11.1 & \\
\hline
\end{tabular}




\begin{tabular}{|c|c|c|c|c|}
\hline Cancer Screening & $\begin{array}{c}\text { Total } \\
\mathrm{N}=6643, \%\end{array}$ & $\begin{array}{c}\text { Asians } \\
\mathrm{N}=620, \%\end{array}$ & $\begin{array}{l}\text { NonAsians } \\
\mathrm{N}=6023, \%\end{array}$ & $\chi^{2}$ \\
\hline$>5$ years ago & 6.7 & 5.0 & 6.8 & \\
\hline Never & 33.3 & 45.0 & 32.6 & 10.2 \\
\hline \multicolumn{5}{|c|}{ Prostate blood test or rectal exam } \\
\hline$<1$ year ago & 46.5 & 38.5 & 47.2 & \\
\hline $1-2$ years ago & 21.3 & 21.1 & 21.3 & \\
\hline $3-5$ years ago & 9.3 & 9.2 & 9.3 & \\
\hline$>5$ years ago & 5.0 & 4.6 & 5.0 & \\
\hline Never & 18.0 & 26.6 & 17.2 & 6.6 \\
\hline
\end{tabular}

Table 4

Predictors of Screening Behavior by Source of Health Information

\begin{tabular}{|c|c|c|c|c|c|}
\hline & $\begin{array}{l}\text { Physical Exam } \\
\text { OR (95\% CI) }\end{array}$ & $\begin{array}{c}\text { Pap test } \\
\text { OR }(95 \% \mathrm{CI})\end{array}$ & $\begin{array}{l}\text { Mammo- } \\
\text { gram } \\
\text { OR (95\% } \\
\text { CI) } \\
\end{array}$ & $\begin{array}{l}\text { Colon cancer } \\
\text { OR }(95 \% \mathrm{CI})\end{array}$ & $\begin{array}{c}\text { Prostate } \\
\text { OR }(95 \% C I)\end{array}$ \\
\hline \multicolumn{6}{|l|}{ Health information source $^{\mathrm{a}}$} \\
\hline From World Wide Web & $\begin{array}{c}.59 \\
(.44, .80)^{*}\end{array}$ & $\begin{array}{c}.66 \\
(.47, .9)^{*}\end{array}$ & $\begin{array}{c}.76 \\
(.6, .9)^{*}\end{array}$ & $\begin{array}{c}.78 \\
(.6,1.0)^{*}\end{array}$ & $\begin{array}{c}.63 \\
(.52, .78)^{*}\end{array}$ \\
\hline $\begin{array}{l}\text { Books or other printed } \\
\text { material }\end{array}$ & $\begin{array}{c}.57 \\
(.45, .73)^{*}\end{array}$ & $\begin{array}{c}.55 \\
(.4, .7)^{*}\end{array}$ & $\begin{array}{c}.54 \\
(.4, .6)^{*}\end{array}$ & $\begin{array}{c}.76 \\
(.6, .9)^{*}\end{array}$ & $\begin{array}{c}1.4 \\
(1.2,1.6)^{*}\end{array}$ \\
\hline $\begin{array}{l}\text { Called Doctor/other HC } \\
\text { provider }\end{array}$ & $\begin{array}{c}.71 \\
(.54, .95)^{*}\end{array}$ & $\begin{array}{c}.63 \\
(.4, .8)^{*}\end{array}$ & $\begin{array}{c}.82 \\
(.7,1.0)^{*}\end{array}$ & $\begin{array}{c}.58 \\
(.5, .7)^{*}\end{array}$ & $\begin{array}{c}.83 \\
(.7,1.0)^{*}\end{array}$ \\
\hline Asked friends or family & $\begin{array}{c}.74 \\
(.58, .95)^{*}\end{array}$ & $\begin{array}{c}.90 \\
(.6,1.2)\end{array}$ & $\begin{array}{c}.64 \\
(.5, .8)^{*}\end{array}$ & $\begin{array}{c}.88 \\
(.7,1.1)\end{array}$ & $\begin{array}{c}1.3 \\
(1.1,1.5)^{*}\end{array}$ \\
\hline Asked Pharmacist & $\begin{array}{c}.74 \\
(.57, .96)^{*}\end{array}$ & $\begin{array}{c}.48 \\
(.3, .7)^{*}\end{array}$ & $\begin{array}{c}.68 \\
(.6, .8)^{*}\end{array}$ & $\begin{array}{c}.78 \\
(.6,1.0)^{*}\end{array}$ & $\begin{array}{c}1.2 \\
(1.0,1.4)^{*}\end{array}$ \\
\hline From Community Health Fair & $\begin{array}{c}.68 \\
(.44,1.07)\end{array}$ & $\begin{array}{c}.92 \\
(.6,1.4)^{*}\end{array}$ & $\begin{array}{c}.65 \\
(.5, .8)^{*}\end{array}$ & $\begin{array}{c}.76 \\
(.56,1.0) \\
\end{array}$ & $\begin{array}{c}1.5 \\
(1.2,2.1)^{*}\end{array}$ \\
\hline \multicolumn{6}{|l|}{$\begin{array}{l}\text { Ease to find information about the } \\
\text { quality of: }\end{array}$} \\
\hline Doctors in community & $\begin{array}{c}.77 \\
(.59,1.0)^{*} \\
\end{array}$ & $\begin{array}{c}.71 \\
(.5,1.0)^{*}\end{array}$ & $\begin{array}{c}.99 \\
(.8,1.2) \\
\end{array}$ & $\begin{array}{c}.67 \\
(.5, .8)^{*}\end{array}$ & $\begin{array}{c}1.1 \\
(.9,1.4) \\
\end{array}$ \\
\hline Health insurance plans & $\begin{array}{c}.65 \\
(.50, .86)^{*}\end{array}$ & $\begin{array}{c}.73 \\
(.5,1.0)\end{array}$ & $\begin{array}{c}1.04 \\
(.9,1.2)\end{array}$ & $\begin{array}{c}.75 \\
(.6, .9)^{*}\end{array}$ & $\begin{array}{c}1.03 \\
(.8,1.2)\end{array}$ \\
\hline Hospitals in community & $\begin{array}{c}.71 \\
(.53, .95)^{*}\end{array}$ & $\begin{array}{c}.72 \\
(.5,1.0)^{*}\end{array}$ & $\begin{array}{c}1.03 \\
(.8,1.3)\end{array}$ & $\begin{array}{c}.73 \\
(.6,1.0)^{*}\end{array}$ & $\begin{array}{c}1.3 \\
(1.1,1.7)^{*}\end{array}$ \\
\hline $\begin{array}{l}\text { Difficulty reading/understanding } \\
\text { information/ booklets at Dr's } \\
\text { office }\end{array}$ & $\begin{array}{c}.46 \\
(.34, .64)^{*}\end{array}$ & $\begin{array}{c}.56 \\
(.4, .8)^{*}\end{array}$ & $\begin{array}{c}.62 \\
(.5, .8)^{*}\end{array}$ & $\begin{array}{c}.72 \\
(.6, .9)\end{array}$ & $\begin{array}{c}.99 \\
(.7,1.3)\end{array}$ \\
\hline $\begin{array}{l}\text { Difficulty speaking with } \\
\text { Physician }^{\mathrm{d}}\end{array}$ & $\begin{array}{c}2.2 \\
(1.15,4.1)^{*}\end{array}$ & $\begin{array}{c}.88 \\
(.3,2.6)\end{array}$ & $\begin{array}{c}.65 \\
(.27,1.6) \\
\end{array}$ & $\begin{array}{c}1.8 \\
(.6,5.1)\end{array}$ & $\begin{array}{c}4.3 \\
(.9,19.4)^{*}\end{array}$ \\
\hline Needed interpreter for Dr. visit & $\begin{array}{c}.89 \\
(.43,1.9)\end{array}$ & $\begin{array}{c}.37 \\
(.1,1.1)\end{array}$ & $\begin{array}{c}.59 \\
(.23,1.6)\end{array}$ & $\begin{array}{c}1.13 \\
(.3,4.1)\end{array}$ & $\begin{array}{c}2.7 \\
(.8,9.1)\end{array}$ \\
\hline
\end{tabular}

Number of respondents varies according to item, NonAsians $(n=6095)$, Asians $(n=627) .{ }^{*} p<.05$ 
Participant access to hospital information led to obtaining most of the tests except mammograms. If respondents reported that it was easy to $\mathrm{read} /$ understand information booklets in a physician's office, they were more likely to get a physical exam, Pap test, and mammogram. Those that reported difficulty speaking with a physician were at 2.2 times at greater risk of never getting a physical exam and 4.3 times at greater risk of never getting a prostate exam.

\section{Discussion}

This study found that Asians used the internet as a source of health information more than nonAsians, which may indicate that the internet is a useful source to influence health behaviors among this population, especially if the information can be tailored to Asians. Asians were less likely to consult with a health care provider or a pharmacist than non-Asians which may indicate tendency to seek out information prior to approaching a health care provider, or they find difficulty approaching health care providers (Saha, Arbelaez, \& Cooper, 2003).

Asians found it more difficult to read or understand information on prescription bottles and in booklets in physician offices than other racial/ethnic groups (National Health Care Disparities Report, 2003), which helps to explain why Asians are less likely to seek health information from health care providers and pharmacists than non-Asians.

Asians preferred to be treated by a racially concordant health care provider which may help reduce communication difficulties. Among those who had difficulty speaking with a physician, Asians were less likely to indicate need for an interpreter, and relied more on family and friends as interpreters, whereas nonAsians relied more on staff persons at the health care facility. Interpreters were less available for Asians than non-Asians. Use of friends and family members is problematic because untrained interpreters often lead to less quality of patient-provider communication (Baker et al., 1996). This study shows that since family members are most often used as interpreters and if these tend to be less available and less accurate than trained interpreters, health care providers should be cautious about using just any available bilingual person, especially children (Ngo-Metzger et al., 2003).

Asians were less likely to have a physical exam and examinations for specific diseases such as cervical cancer, breast cancer, and colon cancer. While cancer is the leading cause of death for Asians, they have the lowest screening rates (American Cancer Society, 2004; Chen \& Koh, 1997). This may reflect Asian attitudes about fatalism toward health. Ill health is associated with misfortune or external forces over which they have no control (Chin \& Bigby, 2003; Rasbridge, 2003). Demographic factors such as a higher education level, having medical insurance, and those who live in the US 10+ years increase the likelihood for screening (American Cancer Society, 2004), however, this was not the case for Asian American women. It is presumed other cultural factors attribute to this disparity. This study found that having access to health information sources such as the World Wide Web, books, a health care provider, and being able to ask a pharmacist increased the odds of getting all the screening tests. Asking family and friends as a source of health information increased the odds of getting an exam for a general physical, breast, and prostate cancer. If participants had access to a health fair, the odds increased for screening for cervical, breast, and prostate cancer.

The odds of never getting a physical exam (OR $=2.2)$ and a prostate exam $(\mathrm{OR}=4.3)$ were increased if participants had difficulty speaking with a physician. While studies (Gandhi et al., 2000) indicate that language is an important barrier to health care access, language is not the only barrier that Asians face. This study found that Asians find it difficult to access other sources of information, prior to meeting with a health care provider.

The study had two limitations. First, it was a cross-sectional study and therefore it is not possible to determine causality. For example, having access to certain sources of health information may be associated with increased 
probability of screening, but it is difficult to ascertain whether access to health sources will lead to improved screening rates. Second, using the category of Asian to include Asian subgroups of Chinese, Vietnamese, and Koreans may mask the disparity within these subgroups. Future studies may explore the differences between racial and ethnic subgroups in the availability and accessibility of various health sources of information among these groups.

The findings in this study indicate differences in sources of health information between Asians and non-Asians, that Asians used family and friends as interpreters for health care services, and that the sources of health information increased the odds that Asians would go for screening. These findings suggest that as a prelude to accessing health care services, consideration should be given to developing sources of health information relevant to Asians so as to enhance their decision making prior to and in conjunction with receiving medical care services. Informed decisions as health care consumers will more likely reduce the health disparity that exists between ethnic/racial groups.

\section{References}

Agency for Healthcare Research and Quality (AHRQ), U.S. Department of Health and Human Services (2003). National Health Care Disparities Report 2003. Retrieved November 20, 2003, from http://www.qualitytools.ahrq.gov/disparitiesreport/download report.aspx

American Cancer Society. (2003). Cancer facts and figures 2004. Retrieved November 20, 2003, from http://www.cancer.org/downloads/STT/CAFF finalPWSecured.pdf

Baker, D. W., Parker, R. M., Williams, M. V., Coates, W. C., \& Pitkin, K. (1996). Use and effectiveness of interpreters in an emergency department. Journal of American Medical Association, 275, 783788.

Burstin, H. R., Lipsitz, S. R., \& Brennan, T. A. (1992). Socioeconomic status and risk for substandard medical care. Journal of the American Medical Association, 268, 2383-2387.

Chen, M. S., \& Koh, H. K. (1997). The need for cancer prevention and control among Asian American and Pacific Islanders. Asian American \& Pacific Islander Journal of Health, 5, 3-6.

Chen, J. Y., Diamant, A. L., Kagawa-Singer, M., Pourat, N., \& Wold, C. (2004). Disaggregating data on Asian and Pacific Islander women to assess cancer screening. American Journal of Preventive Medicine, 27, 139-145.

Chin, J. L., \& Bigby, J. (2003). Care of Asian Americans. In J. Bigby, "Cross-cultural medicine.” Philadelphia: American College of Physicians.

Collins, Hughes, Doty, Ives, Edwards, \& Tenney. (2002). Diverse communities common concerns: Assessing the health quality for minority Americans. New York: The Commonwealth Fund.

Erzinger, S. (1991). Communication between Spanish-speaking patients and their doctors in medical encounters. Culture, Medicine, and Psychiatry, 15, 91.

Frayne, S. M., Burns, R. B., Hardt, E. J., Rosen, A. K., \& Moskowitz, M. A. (1996). The general exclusion of non-English-speaking persons from research. Journal of General Internal Medicine, $11,39-43$.

Gandhi, T. K., Burstin, H. R., Cook, E. F., Puopolo, A. L., Haas, J. S., Brennan, T. A., Bates, D. W. (2000). Drug complications in outpatients. Journal of General Internal Medicine, 15, 149-154.

Ginsberg, C., Martin, V., Andrulis, D., Shaw-Taylor, Y., \& McGregor, C. (1995). Interpretation and translation services in health care: A survey of US public and private teaching hospitals. A National Public Health and Hospitals Institute (NPPHI) Report. Washington (DC).

Haviland, M. G., Morales, L. S., Reise, S. P., \& Hays, R. D. (2003). Do health care ratings differ by race or ethnicity? Joint Commission Journal of Quality and Safety, 29, 134-145.

Institute of Medicine (n.d.). Unequal treatment: Confronting racial and ethnic disparities in healthcare. Retrieved November 20, 2003, from http://www.iom.edu/report.asp?id=4475

Kagawa-Singer, M., \& Pourat, N. (2000). Asian American and Pacific Islander breast and cervical carcinoma screening rates and Healthy People 2000 objectives. Cancer, 89, 696-705. 
Miller, B. A., Kolonel, L. N., Bernstein, L., Young, J. L., Swanson, G. M., West, D., Key, C.R., Liff, J. M., Glover, C. S., \& Alexander, G. A. (Eds.). (1996). Racial/ ethnic patterns of cancer in United States, 1988- 1992. Bethesda, MD: National Cancer Institute, 30-35.

Ngo-Metzger, Q., Massagli, M. P., Clarridge, B. R., Manocchia, M., Davis, R. B., Lezzoni, Li, \& Phillips, R. S. (2003). Linguistic and cultural barriers to care: Perspectives of Chinese and Vietnamese immigrants. Journal of General Internal Medicine, 18, 44-52.

Perez-Stable, E. J., Napoles-Springer, A., \& Miramontes, J. M. (1997). The effect of ethnicity and language on medical outcomes of patients with Hypertension or Diabetes. Medical Care, 35, 1212-1219.

Rasbridge, L. A. Vietnamese-American profile: Asian-American health. Retrived July 21, 2003, from http://www3.baylor.edu/ Charles_Kemp/vietnamese health.htm

Saha, S., \& Hickam, D. H. (2003). Explaining low ratings of patient satisfaction among Asian-Americans. American Journal of Medical Quality, 18, 256-264.

Saha, S., Arbelaez, J. J., \& Cooper, L. A. (2003). Patient-physician relationships and racial disparities in the quality of health care. American Journal of Public Health, 93, 1713-1719.

Sanghavi, G. M., Wee, C. C., McCarthy, E. P., Davis, R. B., Ngo-Metzger, Q., \& Phillips, R. S. (2003). Racial and ethnic disparities in cancer screening: The importance of foreign birth to care. Journal of General Internal Medicine, 18, 1028-1035.

Seijo, R. (1991). Language as a communication barrier in medical care for Latino patients. Hispanic Journal of Behavioral Science, 13, 363.

Williams, M. V., Parker, R. M., Baker, D. W., Parikh, N. S., Pitkin, K., Coates, W. C., \& Nurss, J. R. (1995). Inadequate functional health literacy among patients at two public hospitals. Journal of the American Medical Association, 281, 1677-1682.

\title{
Acknowledgments
}

This publication was supported by NIH-National Cancer Institute's project "ATECAR - Community Cancer Network," at Center for Asian Health, College of Health Professions (Principal Investigator: Grace Ma, Ph.D.)

\author{
Author Information \\ Steven E. Shive, $\mathrm{PhD}$, CHES* \\ Center for Asian Health \\ Temple University \\ and \\ Department of Health \\ East Stroudsburg University \\ DeNike Hall \\ 200 Prospect St. \\ East Stroudsburg, PA 18301-2999 \\ Ph.: 570-422-3330 \\ Fax.: 570-422-3848 \\ E-mail: sshive@po-box.esu.edu \\ Grace X. Ma \\ Department of Public Health \\ Center for Asian Health \\ College of Health Professions \\ Temple University \\ Philadelphia, PA
}


Yin Tan

Center for Asian Health

Department of Public Health

College of Health Professions

Temple University

Philadelphia, PA

Jamil I. Toubbeh

Center for Asian Health

Adjunct Professor

Department of Public Health

College of Health Professions

Temple University

Philadelphia, PA

Lalitha Parameswaran

Center for Asian Health

Department of Public Health

College of Health Professions

Temple University

Philadelphia, PA

Joseph Halowich

Health Faculty

Parkside High School

Salisbury, MD.

* Corresponding author 